\title{
Bloqueo del plano erector de la espina bilateral para esternotomía en cirugía cardiaca: descripción de una serie de casos
}

\author{
David Barra1, Mauricio González², María José Cordero R.²
}

Introducción: Durante los últimos años el manejo intraoperatorio de pacientes sometidos a cirugía cardíaca ha sufrido un cambio fundamental pasando desde usos de altas dosis de opioides a esquemas fast - track con disminución de las dosis de opioides que permitan un proceso de extubación precoz. El bloqueo del plano erector de la espina (ESP) es un bloqueo interfascial guiado por ultrasonido descrito en 2016 para tratar el dolor neuropatico torácico con excelentes resultados. Hasta la fecha ha sido descrito en múltiples serie de casos incluyendo cirugía de tórax, mama y cardiocirugía.

Objetivos: Describir la calidad de analgesia, mediante evaluación del dolor medido por escala visual análoga (EVA), consumo de opioides en las primeras 24 horas y describir el área anestesiada del tórax en paciente sometido a cirugía cardíaca por esternotomía y circulación extracorpórea (CEC) mediante la realización de un bloqueo del plano erector de la espina bilateral.

Métodos: Estudio descriptivo de calidad analgésica en ESP bilateral en 15 pacientes sometidos a cirugía cardíaca y CEC en el Hospital Clínico Universidad de la Chile. Previa obtención del consentimiento informado, bajo monitorización y posición sentada, con transductor lineal en posición longitudinal se identificó el proceso transverso de la quinta vertebra torácica (T5). Una vez identificado, se insertó una aguja stimuplex de $50 \mathrm{~mm}$ en dirección caudal a cefálico, hasta tomar contacto con el proceso trans- verso, se inyectaron $20 \mathrm{ml}$ levobupivacaina al 0,25\% + epinefrina $5 \mathrm{mcg} / \mathrm{ml}$. Luego se repite el bloqueo en el lado contralateral. Tras realizar ambos bloqueo los pacientes fueron colocados en posición supina. Se evaluaron los dermatomos anestesiados a los 10 minutos con test táctiles. El dolor postoperatorio en reposo y movimiento fueron evaluados usando escala visual análoga a las 3 y 12 horas posterior al termino de la cirugía en unidad cuidados intensivos, por residente de anestesiología.

Resultados: 15 pacientes fueron ingresados al protocolo. El $71 \%$ de los pacientes fueron hombres y la edad promedio fue 57 años, $65 \%$ de los pacientes fueron sometidos a cirugía de revascularización coronaria. El $100 \%$ de los pacientes fueron extubados en pabellón y trasladados a unidad coronaria. El 100\% de los pacientes presentaron dolor en reposo EVA $<3$ a las 3 horas en reposo. A las 12 horas el $80 \%$ de los pacientes presentaban dolor EVA 0 en reposo. Los pacientes utilizaron en promedio 1,5 bolos de rescate de fentanilo en las primeras 24 horas, la mayoría durante las primeras 3 horas postoperatorias. El 100\% de los pacientes presentaron bloqueo a nivel de línea axilar anterior y línea medioclavicular y $46 \%$ presentaron bloqueo a nivel paraesternal bilateral.

Conclusión: El bloqueo ESP bilateral realizado a nivel torácico es una alternativa analgésica válida pacientes sometidos a cardiocirugía vía esternotomía presentando un bajo nivel de dolor y de consumo de opioides en el postoperatorio.

Universidad de Chile. Chile.

Anestesiólogo, Departamento Anestesiología y Medicina Perioperatoria Hospital Clínico Universidad de Chile. Chile. 